\title{
Proposing Effective Method to Develop Common Operational Picture in Disaster Response Utilizing Cloud-based Geospatial Infrastructure
}

\author{
Munenari Inoguchi \\ Research Institute for Natural Hazard \& \\ Disaster Recovery, Niigata University, JAPAN \\ Reo Kimura \\ School of Human Science and Environment, \\ University of Hyogo, JAPAN
}

\begin{abstract}
The concept of Mashup, which refers to forming a new service by combining technologies and contents from multiple sources, already exists after Web2.0 in the field of software development; however, its concept was not converted well to social service yet. After Tohoku Earthquake ministries and agencies in Japan found it necessary to organize the pieces of information in a geospatial manner in order to take a comprehensive, panoramic view of situations at the site. We organized the Emergency Mapping Team (EMT) as to be able to gather and analyze the information in the cooperative relationship with government. For example under the serious situation of Fukushima Nuclear accident the EMT organized the layers of the impacted area including the information about indicating the locations of buildings, the evacuation situations in areas under directives to evacuate. Using those layers disaster responders simulated the necessity of human and material resources to support the evacuation operation. In this activity, we have stored the outcomes as spatial layers into the Cloud-based Spatial Data Infrastructure. After this activity at the Cabinet Office, we moved our focus to Iwate Prefecture affected by huge tsunami. Utilizing this infrastructure, the responders at Iwate just added the detail of data which only they gathered through their disaster response, and they could develop the COP in their own view points. In this paper, we discuss the effective method to develop COP utilizing the Cloud-based Spatial Data Infrastructure from the view point of all-Japan to the one of limited affected area.
\end{abstract}

\section{Introduction}

Recent advances in IT technologies, in conjunction with cloud technology, are establishing a foundation for information sharing and information processing. In spite of this, there is almost no mention of the way people use these IT technologies

\author{
Keiko Tamura \\ Risk Management Office, \\ Niigata University, JAPAN \\ Haruo Hayashi \\ Disaster Prevention Research Institute, \\ Kyoto University, JAPAN
}

when IT technology application is discussed. Herein, we explore the issues surrounding the way people use IT technologies through the activities of the Emergency Mapping Team (EMT), which is designed to realize a common operational picture during the field disaster response for the 2011 Great East Japan Earthquake. The challenges and solutions in information processing are considered in order to develop an effective disaster response.

\section{Activities to Obtain a Common Operational Picture}

\subsection{Setting up an Emergency Mapping Team}

It is imperative that we share a common operational picture in order to effectively respond to a disaster. A common operational picture means that we collect, organize, and analyze information from various organizations to draw a whole picture of "what is happening now" and to share that picture with stakeholders. Following the 2007 Niigataken Chuetsu-oki Earthquake, the disaster response headquarters of municipalities of the Niigata Prefecture and the City of Kashiwazaki set up an EMT that uses maps to share a common operational picture among disaster response workers.

The Great East Japan Earthquake on March 11, 2011, was a widespread disaster spanning many prefectures that resulted in about 20,000 deaths and missing people and about 370,000 totally or partially destroyed houses and buildings. The government of Japan faced the task of collecting, organizing, and analyzing information from various organizations to draw a whole picture of "what is happening now." This required the consolidation of fragmented pieces of information obtained through inquiry to various organizations, including tables and data formats, into one dataset. Moreover, each time a piece of critical information that was needed to respond to the disaster was found to be missing, the government had to ask the responding organization. In this instance, the efforts of the government alone were 
not enough to establish a common operational picture. It was necessary to have knowledge and technology from outside of the government. The EMT was set up mainly by researchers, private corporations, and NGOs in response to a request from the Cabinet Office (Disaster Response) on March 12th (the day after the Great East Japan Earthquake). The Emergency Mapping Center (EMC) was set up at the Cabinet Office, and it operated for 48 days until April 28th; it eventually expanded to include 39 organizations.

\subsection{Making a Map by Mashup}

Huge amounts of information were collected from the afflicted areas. Therefore, it was essential to consolidate the collected information, sort it according to its purposes, and convert it into spatial information. In reality, however, these huge amounts of data were not really organized using a standard format, since it required each piece of information to be "organized" during a process from information collection to consolidation. It should be noted that the activities of the EMT were carried out under the concept of Mashup. Mashup refers to forming a new service by combining technologies and contents from multiple sources. This is a feature that functions as if it were a single web service and is formed by combining multiple Application Program Interfaces (API). The EMT collected and organized related information from the Internet, made the maps needed for disaster response in GIS, consolidated them into dynamic maps using the mashup technology over a cloud application, and then made as many unique layers as possible available over the world-wide (Figure 1). Figure 2 shows the top page of the EMT website. This page is composed of static maps and dynamic maps. The selection of a dynamic map, which navigates you to a mashup portal site that allows you to superimpose various information. Using a dynamic map, you can freely superimpose information distributed via domestic and foreign sources, in addition to a layer generated by the EMT, onto one map (Figure 3). Another feature of this website is that it makes it possible to combine information collected before and after the disaster.

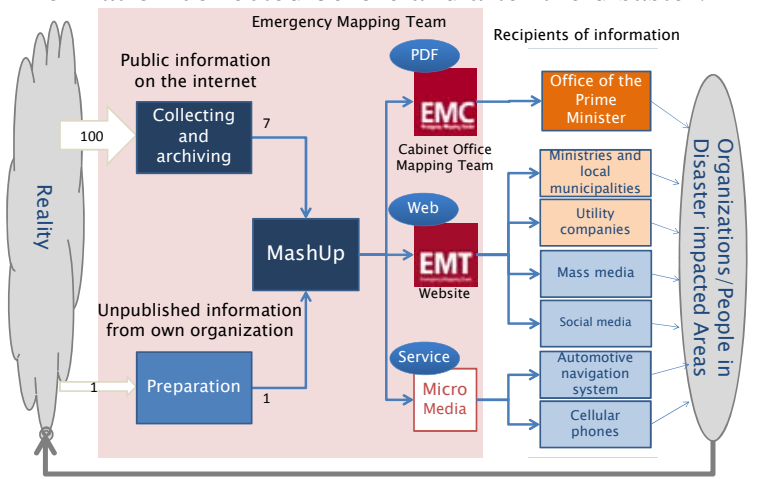

Figure 1. The use of mashup by EMT

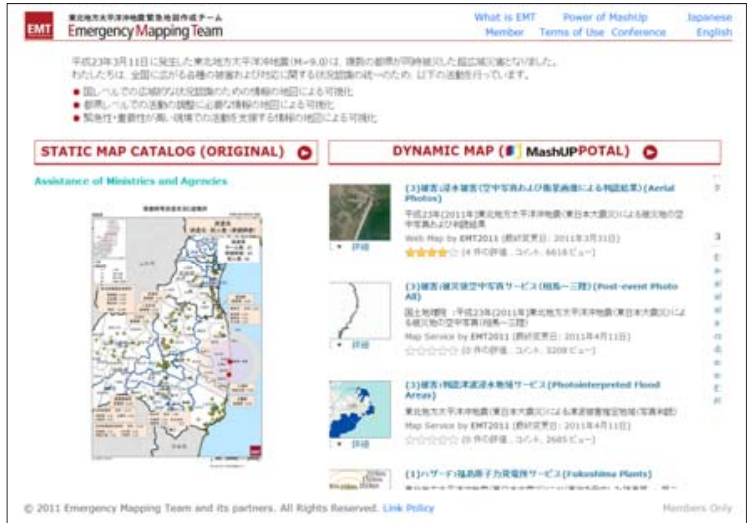

Figure2. The EMT website was composed of static maps and dynamic maps.

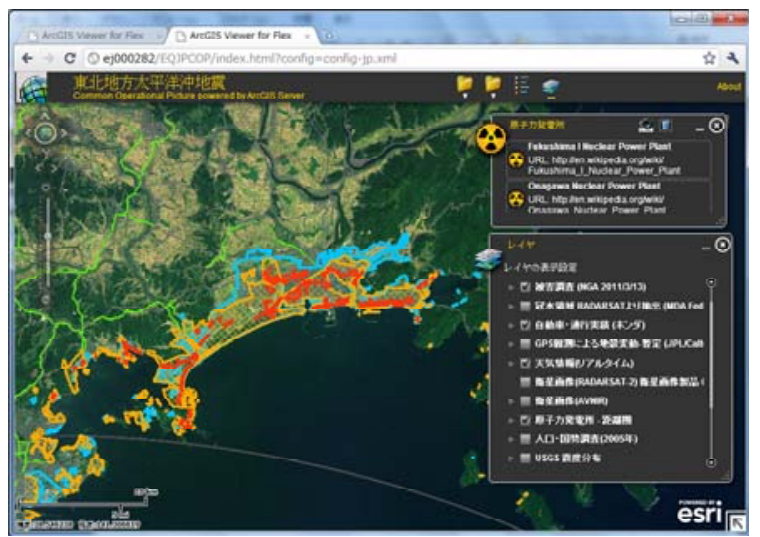

Figure 3. Generating a dynamic map based on a mashed-up portal. The information gap is filled in by mashing-up spatial information from various organizations.

The EMT generated 500 static maps as seen in Table 1. Each map is a realization of a series of processes from spatial analyses using various information gathered from maps requested by government staff. This information was superimposed in a process called mashup. Figure 4 depicts a representative map that was generated 11 days after the disaster. It shows transportation centers and evacuation situations in areas under directives to evacuate or to stay indoors. This map is based on a hazard scenario of "radioactive substances have leaked from the Fukushima Nuclear Plant." Concentric circles are drawn in areas under evacuation advisory and directive, centering on the XY coordinate of the Fukushima Nuclear Plant. In addition, point data for companies were used to indicate the locations of their buildings. The actual number of buildings was calculated by superimposing this data using a spatial statistical method. Locations of evacuation centers as well as the number of evacuees at each center (indicated by numbers next to circles " $\bigcirc$ ") were superimposed with transportation centers, such as airports and harbors, to aid in the decision-making process 
regarding whether it was necessary to close or move existing evacuation centers, or whether to set up new evacuation centers where goods could be transported.

Table 1. Five hundred maps generated by the EMT

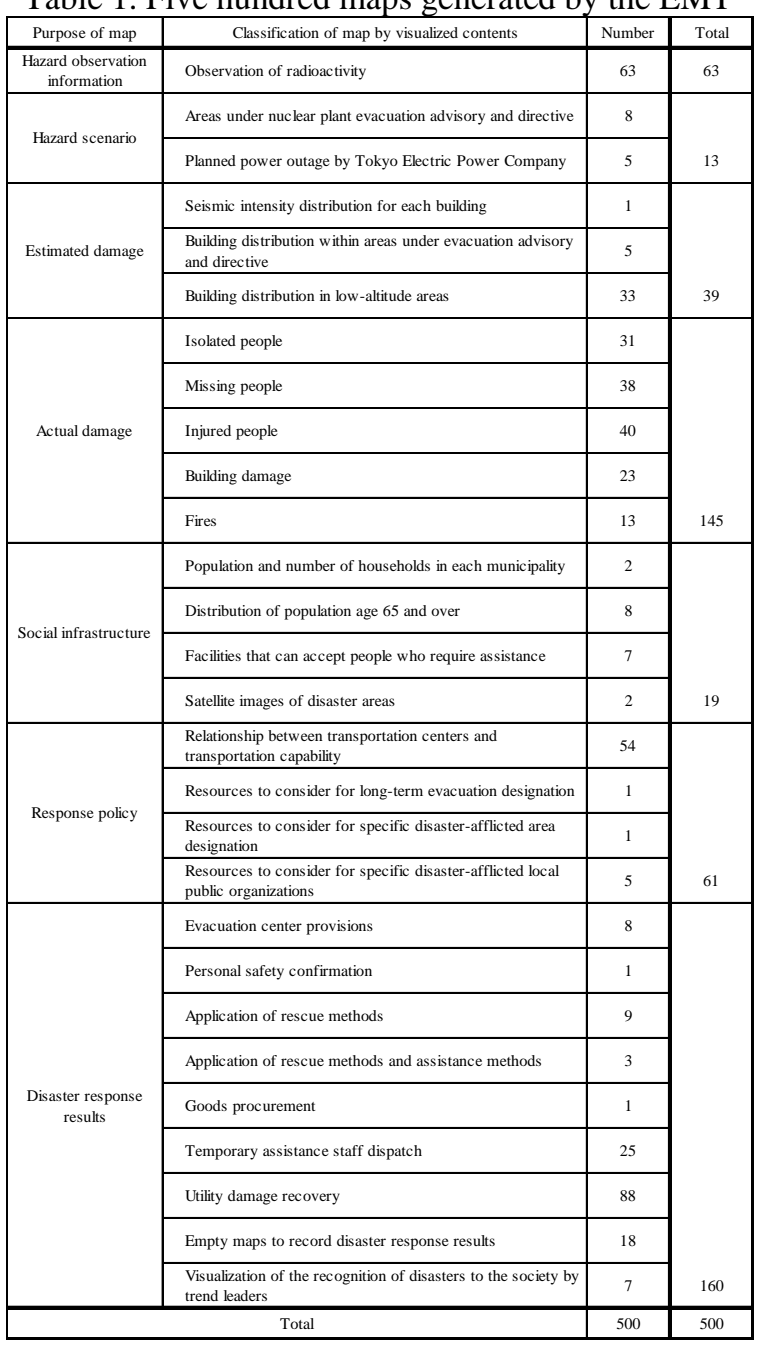

\section{Storing Outcomes into Cloud-based Spatial Data Infrastructure}

In order to cut the cost off to collect or to create the data which are necessary to develop other COPs, we decided to use the Cloud-based Spatial Infrastructure as the platform to store the outcomes of EMT at the Cabinet Office. This infrastructure was composed of three cloud services. First service is Catalog Service, which enables users to search and select maps or geo-processing tools from cloud storage. (Middle of Figure5) Second service is Map Service, which enables users to obtain the maps and layers selected by Catalog Service. (Left-side of Figure5) Third service is Tool Service, which enables users to execute the geo-processing tools selected by Catalog services. (Right-side of Figure5)
At this time, we just thought that we had to share those layers with all kinds of responders, and we stored the 500 maps into the Cloud-based Spatial Data Infrastructure. After this activity, the users, registered to these services as the limited member to use maps and layers, could examine their response browsing the maps and do Mash-up other mapservice or other layers developed by other responders or other agencies in order to develop the COP at latest situation.

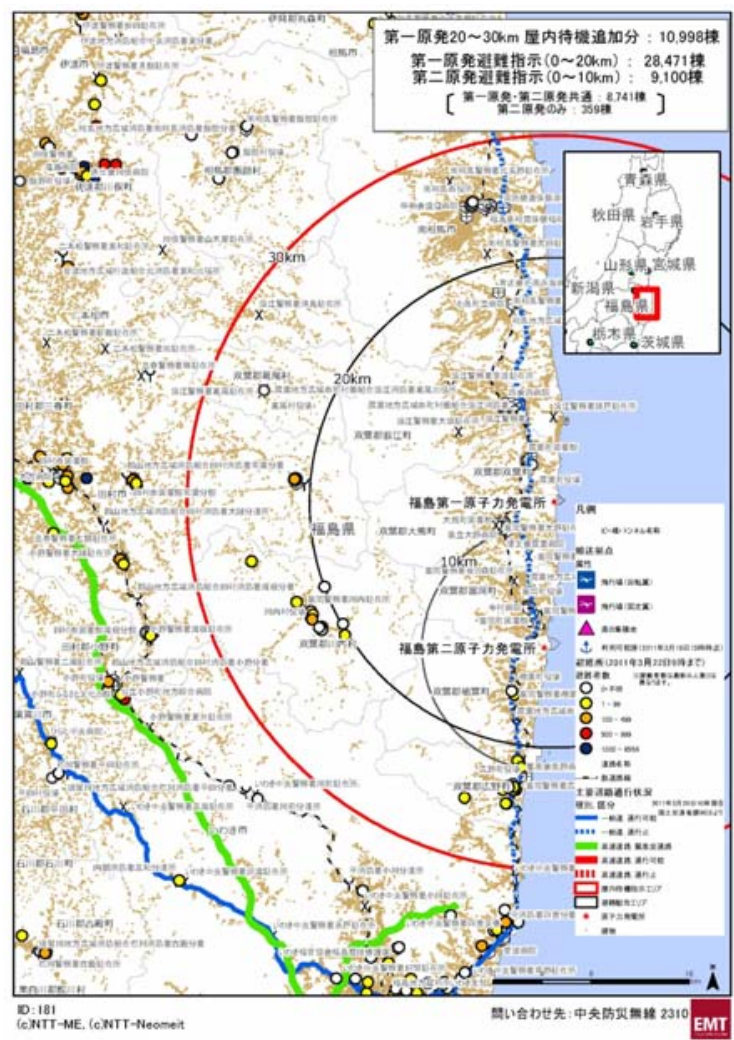

Figure 4. Transportation centers and evacuation situation in the areas near the Fukushima Nuclear Power Plant under directives to evacuate or to stay indoors (Enlarged)

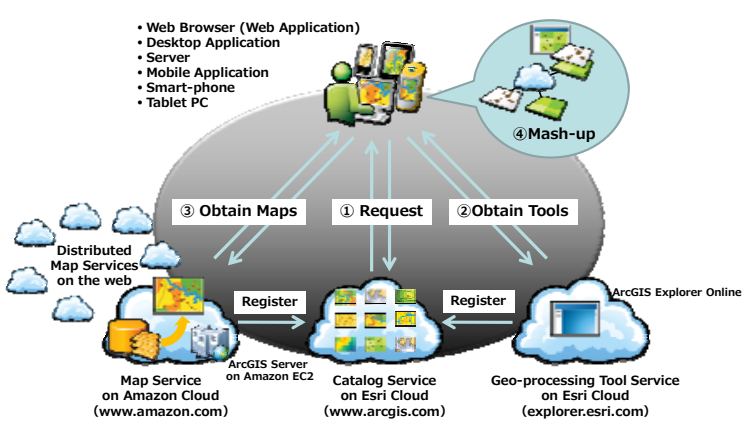

Figure 5. Composition of Cloud-based Spatial Data Infrastructure 


\section{Expanding EMT to Iwate Prefecture}

After the activity of EMT at Cabinet Office for one and half months, we decided to move the activity post to Iwate Prefecture on June $1^{\text {st }}, 2011$. Iwate Prefecture is placed on the northeast area in Japan, and has the long and zigzag shoreline against Pacific Ocean, called "Sanriku shoreline”. At the 2011 Great East Japan Earthquake, this prefecture was attacked by huge tsunami. By this tsunami, 19,199 buildings were totally collapsed, 5,013 buildings were half collapsed, 4,976 persons were dead, and 1,205 persons were disappeared as of September $28^{\text {th }}, 2012$.

Local governments under Iwate Prefecture should respond to those damages soon after huge tsunami attack. When we established EMT at Iwate Prefecture, it had passed about two months from disaster occurrence, so Iwate Prefecture faced on the issues relevant to the recovery and reconstruction process. Against this situation, we decided to support their response by developing COPs. At this time, fortunately, we had already stored the outcomes into the Cloud-based Spatial Infrastructure as spatial layers, and we decided to utilize the stored layers for developing COPs at Iwate Prefecture.

\section{Developing COPs at Iwate Prefecture}

\subsection{Summary of the activity of EMT at Iwate}

As described above, the response phase in Iwate Prefecture had already entered the recovery and reconstruction phase. In this phase, one of most critical issue was how to support the residents in emergency temporary houses. Through the activity of EMT at Cabinet Office, we created the base data about the location and housing-number of emergency temporary houses collected from the web-site of the Ministry of Land, Infrastructure, Transport and Tourism.

At beginning of the activity of EMT at Iwate Prefecture, we refine the data of emergency temporary housings adding the detail data collected from responders in the prefectural government. Then, we registered this data into the Cloud-based Spatial Infrastructure as the spatial layer.

After developing the basic data which is necessary to develop COPs for the responders, we developed 381 types of maps as dynamic maps by doing Mash-up collected data at the prefecture on the basic data (Table 2). 156 maps show the efficiency level of medical services for residents in emergency temporary housings. 90 maps show the risk of landslide to emergency temporary houses. 65 maps shows the efficiency level of welfare and elderly nursing care services for residents with special needs in temporary houses. These three categories occupy the majority of COP maps. In the following section, we describe the detail of those major maps.

Table 2. 381maps were developed by EMT at Iwate

\begin{tabular}{|l|r|}
\hline \multicolumn{1}{|c|}{ Major category } & $\begin{array}{c}\text { Number of } \\
\text { developed maps }\end{array}$ \\
\hline \hline Medical service & 156 \\
\hline Landslide forecast & 90 \\
\hline Welfare and elderly nursing care service & 65 \\
\hline Social statistics & 34 \\
\hline Human and property damage & 13 \\
\hline Temporary emergency house & 11 \\
\hline Evacuation center & 11 \\
\hline Background figure & 1 \\
\hline \hline Total & 381 \\
\hline
\end{tabular}

\subsection{Map of Relationship between Emergency Temporary Houses and Hospitals/Clinics}

After the occurrence of big disaster, public agencies build emerge temporary houses for the victims who lose their houses by the disaster. Those temporary houses usually build in the open space which public agencies own. Most of these open spaces are stood out of downtown area. After the 2011 Great East Japan Earthquake, almost of emergency temporary houses were also built out of downtown area.

Health, Medical Care and Welfare Department of Iwate Prefecture wanted to examine the sufficiency level of health, medical care and welfare services for victims who lived in emergency temporary houses. As this request, we developed the maps of relationship between the emergency temporary houses and hospitals/clinics as shown Figure 6 . Especially, some of hospitals/clinics were affected by tsunami and they could not operate. To develop this map, we had to survey the situation of their operation, and to create the data as spatial layers. Then, by doing Mash-up the situation of hospitals/clinics services operation with the layer of emergency temporary houses, we developed this map to establish COP about the sufficiency level of those services.

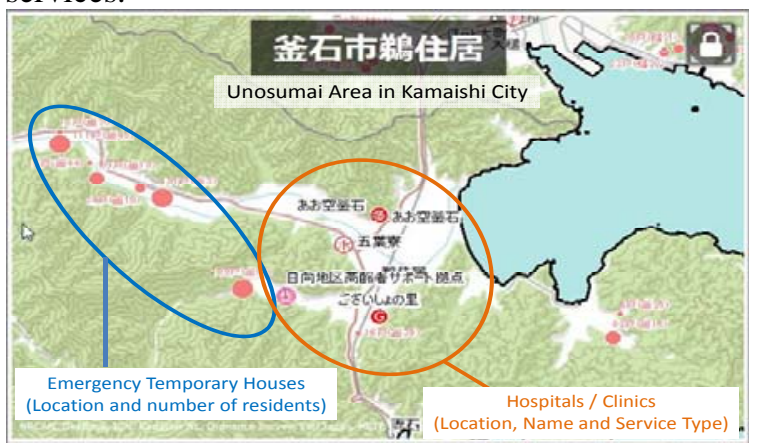

Figure 6. Map of Relationship between Emergency Temporary Houses and Hospitals/Clinics 


\subsection{Map of Relationship between Emergency Temporary Houses and Landslide Risk}

The 2011 Great East Japan Earthquake caused not only huge tsunami and also ground sinking liquefaction. So, most emergency temporary houses were built in mountain area avoiding affected area along shoreline area. This means those houses face to the risk of landslide because most slope of mountain area is steep in Iwate Prefecture.

Against this issue, we developed the maps about relationship between emergency temporary houses and high-risk area of landslide (Figure 7). In figure 7, blue points represent the location and number of households in temporary houses, and red polygons represent the area of steep sloping land. Responders in the prefectural level estimated the landslide risk to residents in emergency temporary housings. However, this map was too severe to be released to residents despite our suggestion.

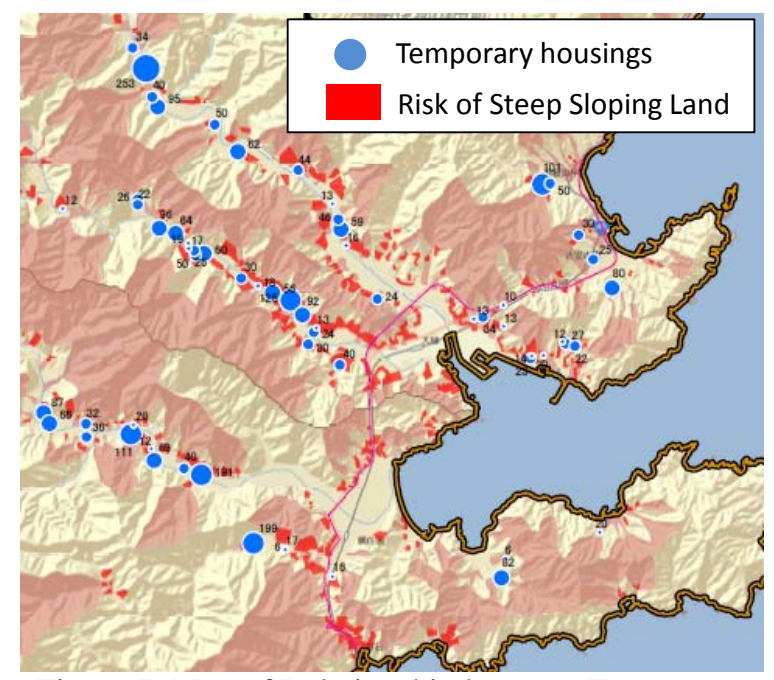

Figure 7. Map of Relationship between Emergency Temporary Houses and Landslide Risk

Because of terrible rainfall on May $4^{\text {th }}, 2012$, some of emergency temporary houses were collapsed. Finally, residents in those collapsed houses had to be moved for evacuation again. If they had estimated the risk of landslide to emergency temporary houses before they were built, this rainfall disaster should be avoided. This activity indicated that it was insufficient only to develop the maps about the actual situation in order to support victims' life reconstruction. Lessons learned from this activity is that we should develop the maps about the estimated situation to avoid new risk in all phase of disaster management.

\section{Discussion}

In this research, we aimed at establishing the effective method to develop COP at huge catastrophe through a case study of 2011 Great East Japan Earthquake. On the next day of disaster occurrence, we established Emergency Mapping Team (EMT) at Cabinet Office in order to support for developing Common Operational Picture (COP), which is necessary for responders to make rational decisions in disaster response.

Because of the features of 2011 Great East Japan Earthquake, which are "wide-area" and "complicated", we had to collect and manage the many kinds of data from many kinds of agencies, and to develop many kinds of maps. Against this issue, we decided to develop COPs in two ways; one is "Static Maps", second is "Dynamic Maps". Especially, dynamic map enables us to do mash-up flexibly on the web with spatial layers which are created by EMT and other agencies. Through the activity of EMT at Cabinet Office, we developed 500 maps for one and half months, and stored outcomes and spatial layers, which maps were composed of, into the Cloud-based Spatial Infrastructure in order to share those maps and layers with other agencies.

After closing the activity of EMT at Cabinet Office, we moved our focus to Iwate Prefecture which is one of affected area by 2011 Great East Japan Earthquake, and we established the activity post of EMT at Iwate Prefecture. At this time, their response phase have already entered recovery and reconstruction phase. Major issue was how to support the residents in emergency temporary houses. Against this issue, we developed 381 maps as COP using layers stored in the Cloud-based Spatial Infrastructure, and layers owned by local responders.

Usually emergency temporary houses are built in the public open space where are out of downtown, so local responders should survey the efficiency level of services which are necessary for victims to live their daily lives. Therefore, we revealed the relationship between the necessary services and the location/number of temporary houses visualizing on the maps. Furthermore, those houses were built in mountain area avoiding affected area in Iwate Prefecture. However, in mountain area, there was much risk of landslide because of steep land slope. In order to estimate the possibility of landslide risk to emergency temporary houses, we revealed the relationship between the area of steep land slope and the location/number of emergency temporary houses. Unfortunately, about one year after disaster occurrence, terrible rainfall attacked the area where emergency temporary houses were built, and some of those houses were collapsed by landslide.

Through our case study at 2011 Great East Japan Earthquake, we proved three issues. One is that it is insufficient only to develop the maps as Static Maps 
for establishing COPs, and so we should also develop the maps as Dynamic Maps for establishing COPs at huge catastrophe. Second is that it is effective way to use the Cloud-based Spatial Data Infrastructure in order to store and share maps and layers among central governments and local governments. Third is that that it is insufficient only to visualize the actual situation on maps, and we should also visualize the estimated situation on maps in order to support for rational decision makings in the response to huge catastrophe.

The result of this research is just one case study at huge catastrophe. Therefore, we have not established the standardized effective method to develop COPs visualizing the situation on maps yet. Against this issue, we have to analyze the work-flow of information process in continuing the activity of EMT. Furthermore, in this research, we have not established effective way to manage the spatial information on cloud-based infrastructure in order to search and retrieve critical or effective information flexibly. We are also planning to continue our research to solve this issue in near future.

\section{Acknowledgements}

Authors would like to thank the following for their support: (1) Ministry of Education, Culture, Sports, Science and Technology, Metropolitan Epicentral Earthquake Disaster Prevention and Disaster Reduction Project, "3. Building System for Wide-Area Risk Management and Disaster Reduction (Principal investigator: Haruo Hayashi, Kyoto University), (2) JST Sakigake "Information and Environment" field "Establishment of Information Consolidation Technology for Prompt Disaster Response Using a Space (Principal investigator: Munenari Inoguchi, Niigata University)," (3) Grant-in-Aid for Young Scientists (Start-up) \#22710161, JSPS (Principal investigator: Munenari Inoguchi, Niigata University).

\section{References}

[1] M. Inoguchi , K. Tamura , T. Furuya ,R. Kimura and H. Hayashi, "Proposal of Effective On-Demand MashUp among Spatial Information from the activity of Emergency Mapping Team - A Case Study of the 2011 off the Pacific Coast of Tohoku Earthquake -,” Journal of Social Safety Science, No. 15, November 2011.

[2] K. Tamura, G. Urakawa, and H. Hayashi, "2007 Emergency Mapping Center Constructing Common Operational Pictures with GIS," Journal of Disaster Research, vol. 5, No. 1, pp. 5-11, February 2010.

[3] M. Inoguchi, K. Tamura, S. Tatsuki, and H. Hayashi, "Realization of Effective Disaster Victim Support through Information Integration and Visualization using GIS,” Third International Conference on Health Informatics, pp.381- 387, January 2010.
[4] Research Center for Natural Hazard \& Disaster Recovery of Niigata University, and Disaster Prevention Research Institute of Kyoto University, "Emergency Mapping Center in Kashiwazaki REPORT,” ISBN:978-4-901768-19-1, pp.96, March 2009.

[5] Disaster Prevention Research Institute of Kyoto University, and Research Center for Natural Hazard \& Disaster Recovery of Niigata University, "Emergency Mapping Center REPORT,” ISBN:978-4-901768-14-6, pp.130, March 2008.

[6] T. Furuya, R. Kimura, M. Inoguchi, K. Tamura, H. Hayashi, "Geospatial Information Improves the Decision-Making Process during the Disaster Response: The Experience of the Emergency Mapping Team in the 2011 off the Pacific Coast of Tohoku Earthquake," Proceedings of $15^{\text {th }}$ World Conference on Earthquake Engineering, pp.8 (Online), September 2012. 